\title{
Gravitational Radiation from Triple Star Systems
}

\author{
Enrico Montanari*, Mirco Calura, and Pierluigi Fortini \\ Department of Physics, University of Ferrara and INFN Sezione di Ferrara, Via Paradiso 12, I-44100 Ferrara, Italy
}

\begin{abstract}
We have studied the main features of the gravitational radiation generated by an astrophysical system constituted of three compact objects attracting one another (only via gravitational interaction) in such a manner that stable orbits do exist. We have limited our analysis to systems that can be treated with perturbative methods. We show the profile of the gravitational waves emitted by such systems. These results can be useful within the framework of the new gravitational astronomy which will be made feasible by means of the new generation of gravitational detectors such as LISA in a no longer far future.
\end{abstract}

PACS number(s): 04.30.Db, 04.20.-q

\section{INTRODUCTION}

A great deal of efforts are aimed at the detection of gravitational waves. Several large research programmes are under development all over the world [1,2]. Consequently the theoretical study of gravitational radiation generated by astrophysical systems is quite a relevant topic. So far this study has been mainly focused on such sources as supernovae explosions, pulsars and compact binaries. In particular these latter sources have been widely investigated throughout all the stages of their evolution [3 6 .

Space based interferometric detectors such as LISA are sensitive to gravitational waves in the frequency band $10^{-4}$ $10^{-1} \mathrm{~Hz}$ [8.9. Many known gravitational waves sources, such as short-period binary star systems, are contained in this bandwidth 10,11. However their waveforms could be biased by spurious effects such as those due to tidal forces, spinning of the stars, post-Newtonian corrections, presence of interstellar medium or of a third body. In order to recognize a gravitational wave from the experimental data, the expected wave form of the signal must be known in a highly accurate way. It is for this reason that many results have been obtained in order to take into account some of the above effects [12 17]. However, to our knowledge, a solution to the problem of the waveform from triple star system is still lacking. In this paper we study the characteristics of the gravitational radiation produced by such a source, emphasizing the substantial differences with the waves originating from a simple binary system, providing a tool in order to recognize astrophysical systems from the analysis of the gravitational signal detected. Future gravitational astronomy will allow to open a new window on the universe besides the usual way to investigate the sky with electromagnetic devices or the relatively new neutrino astronomy (whose birth could be the "neutrino description" of the supernova explosion 1987A 18 21]). In fact gravitational radiation detection could be the only way to discover and study all that astrophysical systems which are not visible with standard methods because they do not emit electromagnetic radiation nor neutrinos. In particular, the existence of a black-hole could be argued by its effect on the gravitational radiation emitted by a binary system. In this paper we show that the gravitational signal of a binary system is modulated by a perturbing third body. We also show that the signal is different in form. Besides, triple star systems formed by a close binary and a third body radiate the same amount of energy of a close binary (or even more) in the region of interest for LISA. This means that this detector can have the same sensitivity for both kinds of system provided that the waveform of the radiation from triple star systems is known. In fact, with this knowledge, one can analyze the two time series that will be the data of LISA, searching for periodic sources not having the typical form of the signal from a binary system [9]. This can be done without any change in the detector project, but only in the data analysis. In this way it would be possible the gravitational analogue of the discovery of triple star systems with electromagnetic timing [22].

\section{THE EQUATIONS OF MOTION}

Let us consider a system composed of three bodies attracting one another via gravitational force and assume that their typical sizes are small compared with their mutual distances. Let $\boldsymbol{r}_{i}$ and $m_{i}$ be the radius vector and the mass

*Electronic address: montanari@fe.infn.it 
of the $i$-th body in a given Galilean reference frame. In order to simplify the problem the following set of variables is introduced:

$$
\begin{aligned}
& \boldsymbol{x}_{(i)}=\boldsymbol{r}_{2}-\boldsymbol{r}_{1} ; \quad \boldsymbol{x}_{(\boldsymbol{o})}=\boldsymbol{r}_{3}-\boldsymbol{r}_{1} ; \quad \boldsymbol{x}_{(\mathrm{cm})}=\frac{m_{1} \boldsymbol{r}_{1}+m_{i} \boldsymbol{r}_{2}+m_{o} \boldsymbol{r}_{3}}{m_{1}+m_{i}+m_{o}} \\
& m_{i}=m_{2} ; \quad m_{o}=m_{3} .
\end{aligned}
$$

where $\boldsymbol{x}_{(\lambda)}(\lambda=i, o)$ are the relative coordinates of the $\lambda$-th body with respect to the first one, while $\boldsymbol{x}_{(\mathrm{cm})}$ are the coordinates of the centre of mass. The equations therefore become (all through the paper $G=c=1$ ):

$$
\begin{aligned}
& \frac{d^{2} \boldsymbol{x}_{(i)}}{d t^{2}}+\frac{\left(m_{1}+m_{i}\right) \boldsymbol{x}_{(i)}}{\left|\boldsymbol{x}_{(i)}\right|^{3}}=\frac{\partial \delta \mathcal{L}_{i}}{\partial \boldsymbol{x}_{(i)}} \\
& \frac{d^{2} \boldsymbol{x}_{(o)}}{d t^{2}}+\frac{\left(m_{1}+m_{o}\right) \boldsymbol{x}_{(o)}}{\left|\boldsymbol{x}_{(o)}\right|^{3}}=\frac{\partial \delta \mathcal{L}_{o}}{\partial \boldsymbol{x}_{(o)}} \\
& \frac{d^{2} \boldsymbol{x}_{(c m)}}{d t^{2}}=0
\end{aligned}
$$

where the so called disturbing functions have been defined

$$
\begin{aligned}
& \delta \mathcal{L}_{i}\left(\boldsymbol{x}_{(i)}, \boldsymbol{x}_{(o)}\right)=m_{o}\left(\frac{1}{\left|\boldsymbol{x}_{(i)}-\boldsymbol{x}_{(o)}\right|}-\frac{\boldsymbol{x}_{(i)} \cdot \boldsymbol{x}_{(o)}}{\left|\boldsymbol{x}_{(o)}\right|^{3}}\right) \\
& \delta \mathcal{L}_{e}\left(\boldsymbol{x}_{(i)}, \boldsymbol{x}_{(o)}\right)=\frac{m_{i}}{m_{o}} \delta \mathcal{L}_{i}\left(\boldsymbol{x}_{(o)}, \boldsymbol{x}_{(i)}\right)
\end{aligned}
$$

We assume that the mutual interaction between $m_{i}$ and $m_{o}$ is a perturbation to their Keplerian motion around $m_{1}$. This is accomplished if the following conditions are fulfilled:

$$
\begin{aligned}
&\left|\boldsymbol{x}_{(i)}\right|<<\left|\boldsymbol{x}_{(\boldsymbol{o})}\right| \\
& \frac{m_{1}+m_{i}}{m_{o}}>\left(\frac{\boldsymbol{x}_{(i)}}{\boldsymbol{x}_{(o)}}\right)^{3} \\
& \frac{m_{i}}{m_{1}+m_{o}}<<\left(\frac{\boldsymbol{x}_{(i)}}{\boldsymbol{x}_{(o)}}\right)^{2}
\end{aligned}
$$

Within the framework of these assumptions it is possible to solve the equations of motion by employing the so called method of variation of parameters in the form due to Lagrange. A complete review of this method, both in its classical formulation and in the post-Newtonian extension, may be found in Refs. 23 25].

In celestial mechanics the usual way to write the solution as a Keplerian motion is [23]:

$$
\begin{aligned}
x_{\lambda}^{1}= & \left(\cos \left(\omega_{\lambda}\right) \cos \left(\Omega_{\lambda}\right)-\cos \left(i_{\lambda}\right) \sin \left(\Omega_{\lambda}\right) \sin \left(\omega_{\lambda}\right)\right) \tilde{x}_{\lambda}^{1}+ \\
& -\left(\sin \left(\omega_{\lambda}\right) \cos \left(\Omega_{\lambda}\right)+\cos \left(i_{\lambda}\right) \sin \left(\Omega_{\lambda}\right) \cos \left(\omega_{\lambda}\right)\right) \tilde{x}_{\lambda}^{2} \\
x_{\lambda}^{2}= & \left(\cos \left(\omega_{\lambda}\right) \sin \left(\Omega_{\lambda}\right)+\cos \left(i_{\lambda}\right) \cos \left(\Omega_{\lambda}\right) \sin \left(\omega_{\lambda}\right)\right) \tilde{x}_{\lambda}^{1}+ \\
& -\left(\sin \left(\omega_{\lambda}\right) \sin \left(\Omega_{\lambda}\right)-\cos \left(i_{\lambda}\right) \cos \left(\Omega_{\lambda}\right) \cos \left(\omega_{\lambda}\right)\right) \tilde{x}_{\lambda}^{2} \\
x_{\lambda}^{3}= & \sin \left(i_{\lambda}\right) \sin \left(\omega_{\lambda}\right) \tilde{x}_{\lambda}^{1}+\sin \left(i_{\lambda}\right) \cos \left(\omega_{\lambda}\right) \tilde{x}_{\lambda}^{2}
\end{aligned}
$$

where

$$
\begin{aligned}
& \tilde{x}_{\lambda}^{1}=a_{\lambda}\left(\cos \left(\eta_{\lambda}\right)-e_{\lambda}\right) \\
& \tilde{x}_{\lambda}^{2}=a_{\lambda} \sqrt{1-e_{\lambda}^{2}} \sin \left(\eta_{\lambda}\right) \\
& M_{\lambda}=n_{\lambda}\left(t-T_{\lambda}\right)=\eta_{\lambda}-e_{\lambda} \sin \left(\eta_{\lambda}\right) \\
& n_{\lambda}=\sqrt{\frac{m_{1}+m_{\lambda}}{a_{\lambda}^{3}}}
\end{aligned}
$$

$(\lambda=i, o)$. Solutions are parameterized by means of the 12 Keplerian elements $a_{\lambda}, e_{\lambda}, M_{\lambda}, w_{\lambda}, \Omega_{\lambda}, i_{\lambda}$ [23,24]. Within the framework of the method of variation of the parameters, the solution to the full problem has the same form as Eqs. (2.10)-(2.16) but with elements slowly varying upon time according to Lagrangian planetary equations [23 24]. 


\section{THE GENERATED GRAVITATIONAL WAVES}

The formal solution-we have reviewed in the previous Section-allows us to achieve the expression of the emitted gravitational wave as a function of the orbital elements and time explicitly. Since orbital parameters are slowly varying, we neglect their time derivative in performing the calculation. First we evaluate the reduced quadrupole moment of the whole system. According to its usual definition [3] we have:

$$
D_{\alpha \beta}=\int \rho\left(3 r^{\alpha} r^{\beta}-r^{2} \delta_{\alpha \beta}\right) d^{3} \boldsymbol{r}
$$

where, in our case,

$$
\rho=\sum_{j=1}^{3} m_{j} \delta\left(\boldsymbol{r}-\boldsymbol{r}_{j}\right)
$$

Substituting eq. (2.1) into the above relations we get

$$
\mathcal{D}^{\alpha \beta}=\mathcal{D}_{(i)}^{\alpha \beta}+\mathcal{D}_{(o)}^{\alpha \beta}+\mathcal{D}_{(i o)}^{\alpha \beta}
$$

where:

$$
\begin{aligned}
& \mathcal{D}_{(\lambda)}^{\alpha \beta}=\mu_{\lambda}\left(3 x_{\lambda}^{\alpha} x_{\lambda}^{\beta}-\boldsymbol{x}_{(\boldsymbol{\lambda})}^{\mathbf{2}} \delta^{\alpha \beta}\right) \quad \lambda=i, o \\
& \mathcal{D}_{(i o)}^{\alpha \beta}=\mu_{i o}\left[3\left(x_{i}^{\alpha} x_{o}^{\beta}+x_{o}^{\alpha} x_{i}^{\beta}\right)-2 \boldsymbol{x}_{(\boldsymbol{i})} \cdot \boldsymbol{x}_{(\boldsymbol{o})} \delta^{\alpha \beta}\right] \\
& \mu_{i}=\frac{m_{i}\left(m_{1}+m_{o}\right)}{m_{1}+m_{i}+m_{o}} \quad \mu_{o}=\frac{m_{o}\left(m_{1}+m_{i}\right)}{m_{1}+m_{i}+m_{o}} \quad \mu_{i o}=\frac{m_{i} m_{o}}{m_{1}+m_{i}+m_{o}}
\end{aligned}
$$

$\alpha$ and $\beta$ run from 1 to 3. The first two terms in Eq. (3.2) are the quadrupole moments of the two binary systems formed by $m_{1}$ and $m_{i}$ (the inner one) and by $m_{1}$ and $m_{o}$ (the outer one); it retains the usual form but orbital elements vary with time. The third term,--an interaction one-is peculiar of the system as a whole. To clarify these points let us consider the components of the quadrupole tensor when eqs. (2.10 2.14) are taken into account. In general one has

$$
\begin{gathered}
\mathcal{D}_{(\lambda)}^{\alpha \beta}=\sum_{i, j} d_{i j}^{\alpha \beta}\left(\omega_{\lambda}, \Omega_{\lambda}, i_{\lambda}\right) \mathcal{Q}^{i j}\left(\tilde{x}_{\lambda}^{1}, \tilde{x}_{\lambda}^{2}\right) \\
\mathcal{D}_{(i o)}^{\alpha \beta}=\sum_{i, j} d_{i j}^{\alpha \beta}\left(\omega_{i}, \Omega_{i}, i_{i}, \omega_{o}, \Omega_{o}, i_{o}\right) \mathcal{Q}_{i o}^{i j}\left(\tilde{x}_{i}^{1}, \tilde{x}_{i}^{2}, \tilde{x}_{o}^{1}, \tilde{x}_{o}^{2}\right)
\end{gathered}
$$

where $d_{i j}^{\alpha \beta}$ are related to the Euler matrices of the rotation linking the intrinsic reference frames of motion to the observer reference frame; $\mathcal{Q}^{i j}$ are the "intrinsic" quadrupole moment coefficients. If the orbital elements did not change, the first expression would be the usual quadrupole tensor of a binary system. The effect of a third body thus induces a modulation of this signal. As for Eq. (3.4), it is a peculiar feature of a three body problem; however the structure of such a term is similar to the preceeding ones.

The knowledge of the quadrupole tensor allows us to write the TT-gauge components of the gravitational waves emitted by the triple system. As it is well known (e.g. [3,26]):

$$
\begin{gathered}
h_{i j}^{T T}=\frac{2}{3|\mathbf{x}|}\left[\left(\frac{d^{2} D_{i j}}{d t^{2}}\right)-n_{i} n^{k}\left(\frac{d^{2} D_{j k}}{d t^{2}}\right)-n_{j} n^{k}\left(\frac{d^{2} D_{i k}}{d t^{2}}\right)+\right. \\
\left.+\frac{1}{2} \delta_{i j} n^{l} n^{k}\left(\frac{d^{2} D_{l k}}{d t^{2}}\right)+\frac{1}{2} n_{i} n_{j} n^{l} n^{k}\left(\frac{d^{2} D_{l k}}{d t^{2}}\right)\right]
\end{gathered}
$$

where $\boldsymbol{n}=\boldsymbol{r} / \boldsymbol{r}$ is the unit vector in the observer direction.

In order to obtain analytic expressions for the metric perturbation, it is simpler to consider the so called true anomaly $\psi_{\lambda}$ instead of the eccentric anomaly $\eta_{\lambda}$. The relation between these two angles is given by [23:

$$
\cos \eta_{\lambda}=\frac{e_{\lambda}+\cos \psi_{\lambda}}{1+e_{\lambda} \cos \psi_{\lambda}}
$$


Starting from the above relation and the definition of $\eta_{\lambda}$ [see eq. (2.15)] it is easy to show that the time derivative of $\psi_{\lambda}$ is given by

$$
\frac{d \psi_{\lambda}}{d t}=\frac{n_{\lambda}\left(1+e_{\lambda} \cos \psi_{\lambda}\right)^{2}}{\sqrt{\left(1-e_{\lambda}^{2}\right)^{3}}}
$$

In order to put the result in a compact form we introduce the Euler Matrices $\boldsymbol{A}_{\lambda}$ with which eqs. 2.10 2.12) can be written as (here ${ }^{t}$ means transposition)

$$
\boldsymbol{x}_{\lambda}=\boldsymbol{A}_{\lambda}^{t} \tilde{\boldsymbol{x}}_{\lambda}
$$

The second derivative of the quadrupole tensor components can be written as ( $\aleph \in\{11,12,0\})$

$$
\begin{aligned}
& \ddot{D}_{(\lambda)}^{i j}=3 \mu_{\lambda} \sum_{\aleph} d_{(\lambda) \aleph}^{i j}\left(\omega_{\lambda}, \Omega_{\lambda}, i_{\lambda}\right) H_{\lambda}^{\aleph}\left(a_{\lambda}, e_{\lambda}, \psi_{\lambda}\right) \\
& \ddot{D}_{(i o)}^{i j}=3 \mu_{i o} \sum_{r, s=1}^{2} d_{(i o) r s}^{i j}\left(\omega_{i}, \omega_{o}, \Omega_{i}, \Omega_{o}, i_{i}, i_{o}\right) H_{(i o)}^{r s}\left(a_{i}, e_{i}, \psi_{i}, a_{o}, e_{o}, \psi_{o}\right)
\end{aligned}
$$

where

$$
\begin{aligned}
& H_{(\lambda)}^{11}=\frac{d^{2}}{d t^{2}}\left(\tilde{x}_{\lambda}^{1} \tilde{x}_{\lambda}^{1}\right)=-\frac{m_{1}+m_{\lambda}}{2 a_{\lambda}\left(1-e_{\lambda}^{2}\right)}\left(3 e_{\lambda} \cos \psi_{\lambda}(t)+4 \cos 2 \psi_{\lambda}(t)+e_{\lambda} \cos 3 \psi_{\lambda}(t)\right) \\
& H_{(\lambda)}^{12}=\frac{d^{2}}{d t^{2}}\left(\tilde{x}_{\lambda}^{1} \tilde{x}_{\lambda}^{2}\right)=-\frac{m_{1}+m_{\lambda}}{a_{\lambda}\left(1-e_{\lambda}^{2}\right)}\left(3 e_{\lambda}+4 \cos \psi_{\lambda}(t)+e_{\lambda} \cos 2 \psi_{\lambda}(t)\right) \sin \psi_{\lambda}(t) \\
& H_{(\lambda)}^{0}=\frac{d^{2}}{d t^{2}}\left(\tilde{r}_{\lambda}^{2}\right)=\frac{2 e_{\lambda}\left(m_{1}+m_{\lambda}\right)}{a_{\lambda}\left(1-e_{\lambda}^{2}\right)}\left(e_{\lambda}+\cos \psi_{\lambda}(t)\right) \\
& H_{(i o)}^{11}=\frac{d^{2}}{d t^{2}}\left(\tilde{x}_{i}^{1} \tilde{x}_{o}^{1}\right)=-\frac{a_{i}\left(1-e_{i}^{2}\right)\left(m_{1}+m_{o}\right) \cos \left(\psi_{o}(t)\right)\left(1+e_{o} \cos \left(\psi_{o}(t)\right)\right)^{2} \cos \left(\psi_{i}(t)\right)}{a_{o}^{2}\left(1-e_{o}^{2}\right)^{2}\left(1+e_{i} \cos \left(\psi_{i}(t)\right)\right)}+ \\
& -\frac{a_{o}\left(1-e_{o}^{2}\right)\left(m_{1}+m_{i}\right) \cos \left(\psi_{o}(t)\right) \cos \left(\psi_{i}(t)\right)\left(1+e_{i} \cos \left(\psi_{i}(t)\right)\right)^{2}}{a_{i}^{2}\left(1-e_{i}^{2}\right)^{2}\left(1+e_{o} \cos \left(\psi_{o}(t)\right)\right)}+ \\
& +\frac{2 \sqrt{m_{1}+m_{i}} \sqrt{m_{1}+m_{o}} \sin \left(\psi_{o}(t)\right) \sin \left(\psi_{i}(t)\right)}{\sqrt{a_{o}\left(1-e_{o}^{2}\right)} \sqrt{a_{i}\left(1-e_{i}^{2}\right)}} \\
& H_{(i o)}^{12}=\frac{d^{2}}{d t^{2}}\left(\tilde{x}_{i}^{1} \tilde{x}_{o}^{2}\right)=-\frac{a_{i}\left(1-e_{i}{ }^{2}\right)\left(m_{1}+m_{o}\right)\left(1+e_{o} \cos \left(\psi_{o}(t)\right)\right)^{2} \cos \left(\psi_{i}(t)\right) \sin \left(\psi_{o}(t)\right)}{a_{o}^{2}\left(1-e_{o}^{2}\right)^{2}\left(1+e_{i} \cos \left(\psi_{i}(t)\right)\right)}+ \\
& -\frac{a_{o}\left(1-e_{o}^{2}\right)\left(m_{1}+m_{i}\right) \cos \left(\psi_{i}(t)\right)\left(1+e_{i} \cos \left(\psi_{i}(t)\right)\right)^{2} \sin \left(\psi_{o}(t)\right)}{a_{i}{ }^{2}\left(1-e_{i}{ }^{2}\right)^{2}\left(1+e_{o} \cos \left(\psi_{o}(t)\right)\right)}+ \\
& -\frac{2 \sqrt{m_{1}+m_{i}} \sqrt{m_{1}+m_{o}}\left(e_{o}+\cos \left(\psi_{o}(t)\right)\right) \sin \left(\psi_{i}(t)\right)}{\sqrt{a_{o}\left(1-e_{o}^{2}\right)} \sqrt{a_{i}\left(1-e_{i}^{2}\right)}} \\
& H_{(i o)}^{21}=\frac{d^{2}}{d t^{2}}\left(\tilde{x}_{i}^{2} \tilde{x}_{o}^{1}\right)=-\frac{2 \sqrt{m_{1}+m_{i}} \sqrt{m_{1}+m_{o}}\left(e_{i}+\cos \left(\psi_{i}(t)\right)\right) \sin \left(\psi_{o}(t)\right)}{\sqrt{a_{o}\left(1-e_{o}^{2}\right)} \sqrt{a_{i}\left(1-e_{i}^{2}\right)}}+ \\
& -\frac{a_{i}\left(1-e_{i}^{2}\right)\left(m_{1}+m_{o}\right) \cos \left(\psi_{o}(t)\right)\left(1+e_{o} \cos \left(\psi_{o}(t)\right)\right)^{2} \sin \left(\psi_{i}(t)\right)}{a_{o}^{2}\left(1-e_{o}^{2}\right)^{2}\left(1+e_{i} \cos \left(\psi_{i}(t)\right)\right)}+ \\
& -\frac{a_{o}\left(1-e_{o}^{2}\right)\left(m_{1}+m_{i}\right) \cos \left(\psi_{o}(t)\right)\left(1+e_{i} \cos \left(\psi_{i}(t)\right)\right)^{2} \sin \left(\psi_{i}(t)\right)}{a_{i}{ }^{2}\left(1-e_{i}{ }^{2}\right)^{2}\left(1+e_{o} \cos \left(\psi_{o}(t)\right)\right)}
\end{aligned}
$$




$$
\begin{aligned}
& H_{(i o)}^{22}=\frac{d^{2}}{d t^{2}}\left(\tilde{x}_{i}^{2} \tilde{x}_{o}^{2}\right)=\frac{2 \sqrt{m_{1}+m_{i}} \sqrt{m_{1}+m_{o}}\left(e_{o}+\cos \left(\psi_{o}(t)\right)\right)\left(e_{i}+\cos \left(\psi_{i}(t)\right)\right)}{\sqrt{a_{o}\left(1-e_{o}^{2}\right)} \sqrt{a_{i}\left(1-e_{i}^{2}\right)}}+ \\
& -\frac{a_{i}\left(1-e_{i}^{2}\right)\left(m_{1}+m_{o}\right)\left(1+e_{o} \cos \left(\psi_{o}(t)\right)\right)^{2} \sin \left(\psi_{o}(t)\right) \sin \left(\psi_{i}(t)\right)}{a_{o}^{2}\left(1-e_{o}^{2}\right)^{2}\left(1+e_{i} \cos \left(\psi_{i}(t)\right)\right)}+ \\
& -\frac{a_{o}\left(1-e_{o}^{2}\right)\left(m_{1}+m_{i}\right)\left(1+e_{i} \cos \left(\psi_{i}(t)\right)\right)^{2} \sin \left(\psi_{o}(t)\right) \sin \left(\psi_{i}(t)\right)}{a_{i}{ }^{2}\left(1-e_{i}^{2}\right)^{2}\left(1+e_{o} \cos \left(\psi_{o}(t)\right)\right)} .
\end{aligned}
$$

The above coefficients depend only upon the parameters $a_{\lambda}, e_{\lambda}$ and $M_{\lambda}$, while

$$
\begin{aligned}
d_{(\lambda) 11}^{i j} & =A_{(\lambda) 1}{ }^{i} A_{(\lambda) 1}{ }^{j}-A_{(\lambda) 2}{ }^{i} A_{(\lambda) 2}{ }^{j} \\
d_{(\lambda) 12}^{i j} & =A_{(\lambda) 1}{ }^{i} A_{(\lambda) 2}{ }^{j}+A_{(\lambda) 2}{ }^{i} A_{(\lambda) 1}{ }^{j} \\
d_{(\lambda) 0}^{i j} & =A_{(\lambda) 2}{ }^{i} A_{(\lambda) 2}{ }^{j}-\frac{1}{3} \delta^{i j} \\
d_{(i o) r s}^{a b} & =3\left(A_{(i) r}{ }^{a} A_{(o) s}{ }^{b}+A_{(i) r}{ }^{b} A_{(o) s}{ }^{a}\right)-2 \delta^{a b} \sum_{j} A_{(i) r}{ }^{j} A_{(o) s j}
\end{aligned}
$$

depend only upon the Euler angles $\omega_{\lambda}, \Omega_{\lambda}$ and $i_{\lambda}$. The TT-gauge components of the gravitational field given in Eq. (3.5) can then be written as

$$
h_{i j}^{(T T)}=h_{i j}^{(i)}+h_{i j}^{(o)}+h_{i j}^{(i o)}
$$

where

$$
\begin{aligned}
& h_{i j}^{(\lambda)}=\sum_{\aleph} c_{(\lambda) \aleph}^{i j}\left(\omega_{\lambda}, \Omega_{\lambda}, i_{\lambda}, \boldsymbol{n}\right) H_{\lambda}^{\aleph}\left(a_{\lambda}, e_{\lambda}, \psi_{\lambda}\right) \\
& h_{i j}^{(i o)}=\sum_{r, s=1}^{2} c_{(i o) r s}^{i j}\left(\omega_{i}, \omega_{o}, \Omega_{i}, \Omega_{o}, i_{i}, i_{o}, \boldsymbol{n}\right) H_{(i o)}^{r s}\left(a_{i}, e_{i}, \psi_{i}, a_{o}, e_{o}, \psi_{o}\right)
\end{aligned}
$$

The values of the coefficients $c_{(\lambda) \aleph}^{i j}\left(\omega_{\lambda}, \Omega_{\lambda}, i_{\lambda}, \boldsymbol{n}\right)$ and $c_{(i o) r s}^{i j}\left(\omega_{i}, \omega_{o}, \Omega_{i}, \Omega_{o}, i_{i}, i_{o}, \boldsymbol{n}\right)$, connecting the Euler angles with the observer-source direction, are given in appendix.

The signal is therefore the sum of three terms: two binary-like and an interaction one. As for the first two terms, they are equal in form to the signal emitted by two binary systems 《传; however the orbital elements are not constants; this fact results in a modulation of the signal. The third term in Eq. (3.20) is a peculiarity arising from the interaction between these two binary systems. Therefore the signal is described by a Fourier series with slowly variable coefficients. Within the framework of conditions (2.8)-(2.9) the interaction term is of the same order of or greater than the inner one. Therefore, when the outer signal can be neglected (this may happen, for instance, if the sensitivity of the detector near $n_{o}$ is negligibly small; see following section), the signal form is different from that one emitted by a simple two-body system.

\section{APPLICATIONS}

The time variation of orbital elements, induced by the disturbing functions, is very slow and it is quite difficult to observe within an interval of time which is of the same order of magnitude of the smaller unperturbed period involved. Therefore we are interested in the so called "secular" variation of the parameters. For the sake of simplicity we set $i_{i}=i_{o}=i$, that is to say we assume the two orbital planes to coincide. Consequently we have $\Omega_{i}=\Omega_{o}=\Omega$ (because there is only one line of nodes). As we are only interested in secular variations of the elements, the disturbing functions (2.5) and (2.6) may be averaged to obtain:

$$
\begin{gathered}
\left\langle\delta \mathcal{L}_{i}\right\rangle=\frac{m_{o}}{a_{o}}\left(1+\frac{a_{i}^{2}}{4 a_{o}^{2}} \frac{1+\frac{3}{2} e_{i}^{2}}{\left(1-e_{o}^{2}\right)^{\frac{3}{2}}}\right) \\
\left\langle\delta \mathcal{L}_{o}\right\rangle=\frac{m_{i}}{m_{o}}\left\langle\delta \mathcal{L}_{i}\right\rangle
\end{gathered}
$$


This result is exact in both $e_{i}$ and $e_{o}$; see Ref. 24] for an expansion up to second order in both eccentricities. By using Lagrange equations [23,24], up to first order of approximation, one gets:

$$
\begin{aligned}
& \tilde{\omega}_{i}=\nu_{i} t+\tilde{\omega}_{i 0} \\
& \tilde{\omega}_{o}=\nu_{o} t+\tilde{\omega}_{o 0} \\
& M_{i}=N_{i} t+\sigma_{i} \\
& M_{o}=N_{o} t+\sigma_{o}
\end{aligned}
$$

where we have put $\tilde{\omega}_{i}=\omega_{i}+\Omega_{i}, \tilde{\omega}_{o}=\omega_{o}+\Omega_{o} . \tilde{\omega}_{i 0}, \tilde{\omega}_{o 0}, \sigma_{i}$ and $\sigma_{o}$ are constants which are determined once the initial conditions are given, while $\nu_{\lambda}$ are the rates of periastron precessions. Their values are given by the following relations:

$$
\begin{aligned}
& \nu_{i}=\frac{3 m_{o}}{4 n_{i} a_{o}^{3}} \frac{\sqrt{1-e_{i}^{2}}}{\sqrt{\left(1-e_{o}^{2}\right)^{3}}} \\
& \nu_{o}=\frac{3 m_{i} a_{i}^{2}}{4 a_{o}^{5} n_{o}} \frac{\left(1+\frac{3}{2} e_{i}^{2}\right)}{1-e_{o}^{2}} \\
& N_{i}=n_{i}\left[1-\frac{7 m_{o}}{4 a_{o}^{3} n_{i}^{2}} \frac{\left(1+\frac{3}{7} e_{i}^{2}\right)}{\sqrt{\left(1-e_{o}^{2}\right)^{3}}}\right] \\
& N_{o}=n_{o}\left[1+\frac{2 m_{i}}{a_{o}^{3} n_{o}^{2}}+\frac{3 m_{i} a_{i}^{2}}{4 a_{o}^{5} n_{o}^{2}} \frac{\left(1+\frac{3}{2} e_{i}^{2}\right)}{\sqrt{\left(1-e_{o}^{2}\right)^{3}}}\right]
\end{aligned}
$$

By the way, we point out that the averaged Lagrangian given in Ref. [24], p. 320, does not yield the correct secular variation up to second order in eccentricities. In fact, inspection of Lagrangian equations shows that the averaged disturbing function should be expanded up to second order in both eccentricities, without neglecting terms proportional to $e_{i}^{2} e_{o}^{2}$.

Summing up, the secular effects of the interaction between $m_{i}$ and $m_{o}$ are a change in the frequency of the unperturbed motion and appearance of two new frequencies related to the periastron precession.

In the framework of assumptions (2.8)-(2.9), which are the conditions for the perturbative approach to be allowed, we consider two possible systems. The first one is a close binary system orbiting around a third body whose mass is much greater than the total mass of the other two bodies. In other words the order of magnitude of the binary system size is much smaller than the mean distance between its centre of mass and the third body (the so called "lunar case").

The second system is a body orbiting around a close binary system whose total mass is much greater than that of the far body (the so called "solar system case").

\section{A. First case}

Let us consider two stars whose masses are $m_{1}$ and $m_{i}<<m_{1}$ that, besides a motion around their centre of mass, are orbiting around a third star with a mass $m_{o}>>m_{1}+m_{i}$. This two Keplerian orbits, called respectively inner and outer orbit, are perturbed by the disturbing functions (4.1)-(4.2). The effect of these perturbative terms, as we have already seen, is to cause a variation of the elements of the unperturbed Keplerian motions.

In order to show the peculiarity of the gravitational signal in such a situation we consider the following example in which we have assigned: $m_{1}=2 \cdot 10^{31} \mathrm{Kg} \approx 10 \mathrm{~m}_{\odot}, m_{i}=2 \cdot 10^{29} \mathrm{Kg} \approx 10^{-1} \mathrm{~m}_{\odot}, m_{o}=2 \cdot 10^{32} \mathrm{Kg} \approx 100 \mathrm{~m}_{\odot}$, $a_{i}=10^{9} m, a_{o}=10 a_{i}|\boldsymbol{R}|=10 \mathrm{kpc}, e_{i}=e_{e}=0.25, i=0, \Omega=0, \boldsymbol{n}=(0,0,1), \tilde{\omega}_{i 0}=1.1, \tilde{\omega}_{o 0}=0.4, \sigma_{i}=0.3$ and $\sigma_{o}=0.1$. It could be formed by a neutron star close to a black-hole a solar diameter apart, under the influence of a farther more massive black-hole.

The motion of this system could be viewed, in zero-th order of approximation, as the Keplerian motion of the centre of mass of the two closest stars around the far body and the relative motion, Keplerian too, of the binary system. The perturbation, due to the interaction among the three bodies, is therefore described by the variation of the elements of these two Keplerian orbits. The effect results in the appearance of two new frequencies (the rates of periastron precession, see Eqs, (4.7) and (4.8) ) in the gravitational signal emitted besides the two Keplerian ones (see Eqs (2.16), (4.9), and (4.10)). In this case we find 


$$
\begin{array}{rlrl}
N_{i} & =1.14 \times 10^{-3} \frac{\mathrm{rad}}{\mathrm{s}} ; & N_{o} & =1.21 \times 10^{-4} \frac{\mathrm{rad}}{\mathrm{s}} ; \\
\nu_{i} & =9.19 \times 10^{-6} \frac{\mathrm{rad}}{\mathrm{s}} ; & \nu_{o}=9.64 \times 10^{-10} \frac{\mathrm{rad}}{\mathrm{s}}
\end{array}
$$

We notice that a similar effect is due to the 1PN perturbation of the inner orbit; in this case the 1PN frequency is given by [3]:

$$
\nu_{i}^{1 P N}=\frac{3\left(m_{1}+m_{i}\right)}{a_{i}\left(1-e_{i}^{2}\right)} n_{i}=5.56 \times 10^{-8} \mathrm{rad} / \mathrm{s} .
$$

Since our treatment of the phenomena is a classical one, its validity holds for observational times smaller than $2 \pi / \nu_{i}^{1 P N} \simeq 4 y$. Therefore, for this kind of systems, only frequencies greater than this one, that is to say $n_{i}, n_{o}$ and $\nu_{i}$ could be appreciate.

In the previous section we have shown that the gravitational wave emitted is the sum of three terms: the first (second) one is due to the inner (outer) motion and involves two frequencies, that is to say $N_{i}\left(N_{o}\right)$ and $\nu_{i}\left(\nu_{o}\right)$; the last one is an interaction term which depends on all the four frequencies. From what was said it is clear that for the outer term the modulation of signal cannot be observed, while, as far as the interaction term is concerned, only two frequencies yield an observable modulation.

A gravitational detector presents a sensitivity which depends on the frequency. If we consider a detector for which the ratio between sensitivity near $N_{o}$ and near $N_{i}$ is negligible small (such an assumption is quite realistic for LISA, see for instance [8]) then the detectable wave profile is merely the sum of the inner and interaction terms even if the greater amplitude is associated with the outer motion (as it is in this case). The output results in a signal with frequency $N_{i}$ whose amplitude is modulated by the two frequencies $N_{o}$ and $\nu_{i}$ (inner precession rate).

In the figures below (figs. 1-3) we show the gravitational wave form for the inner term, the interaction one and their sum. Time is given in units of the period of the inner orbit.

\section{B. Second Case}

The second kind of triple-system we consider, involves a star of mass $m_{1}$ and other two bodies (whose masses are $m_{i}$ and $m_{o}$ ) moving around it; in addition the following relation holds: $m_{1} \gg m_{o} \gg m_{i}$. We assume that the motion of both $m_{i}$ and $m_{o}$ around the central body $m_{1}$ is a Keplerian one with orbital elements varying with time because of the mutual interaction between the two lighter bodies.

Now, as it has already be done in the previous case, we study the gravitational waves generated by such a kind of system in a particular case. In doing so we have set: $m_{1}=2 \times 10^{32} \mathrm{Kg} \approx 10^{2} \mathrm{~m}_{\odot}, m_{i}=2 \times 10^{29} \mathrm{Kg} \approx 10^{-1} m_{\odot}$, $m_{o}=2 \times 10^{31} \mathrm{Kg} \approx 10 m_{\odot}, a_{i}=4 \times 10^{9} \mathrm{~m}, a_{o}=10 a_{i},|\boldsymbol{R}|=10 \mathrm{kpc} e_{i}=e_{e}=0.25, i=0, \Omega=0, \boldsymbol{n}=(0,0,1)$, $\tilde{\omega}_{i 0}=1.1, \tilde{\omega}_{o 0}=0.4, \sigma_{i}=0.3$ and $\sigma_{o}=0.1$. In this case the two Keplerian frequencies of the inner and outer orbit $\left(N_{i}\right.$ and $N_{o}$ respectively) are:

$$
N_{i}=4.57 \times 10^{-4} \frac{\mathrm{rad}}{\mathrm{s}} ; \quad N_{o}=1.52 \times 10^{-5} \frac{\mathrm{rad}}{\mathrm{s}}
$$

while the two periastron precession rates are:

$$
\nu_{i}=3.65 \times 10^{-8} \frac{\mathrm{rad}}{\mathrm{s}} ; \quad \nu_{o}=1.20 \times 10^{-10} \frac{\mathrm{rad}}{\mathrm{s}}
$$

The frequency involved in the $1 \mathrm{PN}$ perturbation of the inner orbit is:

$$
\nu_{i}^{1 P N}=\frac{3\left(m_{1}+m_{i}\right)}{a_{i}\left(1-e_{i}^{2}\right)} n_{i} \approx 5.42 \times 10^{-8} \frac{r a d}{s}
$$

From (4.14) it follows that the validity of our results holds for times smaller than $2 \pi / \nu_{i}^{1 P N}=1.2 \times 10^{8} \sec \approx 4 y$, therefore we can not appreciate the frequencies $\nu_{i}$ and $\nu_{o}$. For this reason the detectable frequencies of the signal are the only Keplerian ones; it follows that the inner and outer term of the wave profile are not modulated, while the interaction term is modulated by only one frequency.

As in the first case, we show graphically (figs. 4-5) the gravitational wave form; the observation time is measured in units of the periods of the inner orbit. Also in this case if the ratio between the detector sensitivities near $N_{o}$ and $N_{i}$ is negligible small, then the detectable signal is the sum of the inner and interaction terms, which gives a signal with frequency $N_{i}$ and amplitude modulated by the outer frequency $N_{o}$ (fig. 6). 


\section{CONCLUSIONS}

In this paper we have studied the gravitational radiation emitted by triple-star systems.

We have shown that the signal emitted by a triple star system such as that envisaged here is the sum of three terms. Two of them are equal in form to the signals from two binary systems, each of them modulated by the secular variation of the orbital elements. The last term originates in the three-body interaction, not having a two-body counterpart. It has a fundamental frequency near $n_{i}$ (angular frequency of inner motion) and it is modulated by $n_{o}$ (angular frequency of outer motion) and by the secular variation of orbital elements. If $n_{i}$ is the only frequency in the range of detector sensitivity (for LISA such an assumption is quite realistic) then a close binary system in interaction with a third body, in the assumptions made through the paper, gives rise to an output signal which is different in form from the signal belonging to an isolated two-body system.

If the inner motion is in the region of this detector sensitivity, the waveform could be experimentally extracted by the two time series which will be the data of the space based interferometer LISA. Therefore space based interferometers able to detect gravitational radiation from galactic close binary systems will make possible the discovery and study of eventual three-body systems, without any change in their planned design.

In order to test the applicability of this approach we have considered two particular cases. We have found that, because of the Newtonian approximation we used, there could be systems of interest for which this treatment is enough precise to answer for three (first case) or two (last case) modulating frequencies. In this paper we have assumed the post-newtonian effects involved in motion of the bodies to be negligible. Actually, were two of the three bodies very close to each other, such effects would become too important to be neglected. In this case our classical perturbative approach to the motion should be replaced by its post-Newtonian extension (see [25], where relativistic corrections in a simple three-body system are investigated).

\section{ACKNOWLEDGMENTS}

The authors are thankful to G. Corbelli, D. Boccaletti, and A. Guarnieri for useful discussions, and to V. Guidi for reading of the manuscript.

\section{APPENDIX:}

In the following we give the values of the coefficients $c_{(\lambda) \aleph}^{i j}\left(\omega_{\lambda}, \Omega_{\lambda}, i_{\lambda}, \boldsymbol{n}\right)$ and $c_{(i o) r s}^{i j}\left(\omega_{i}, \omega_{o}, \Omega_{i}, \Omega_{o}, i_{i}, i_{o}, \boldsymbol{n}\right)$ which enter in eqs. (3.21)-(3.22). For simplicity we have omitted lower indices, being understood that the following relations hold for every value of them.

$$
\begin{aligned}
c^{11} & =\left(1-\frac{3}{2} n_{1}^{2}+\frac{1}{2} n_{1}^{4}-\frac{1}{2} n_{2}^{2}-\frac{1}{2} n_{1}^{2} n_{2}^{2}\right) d^{11}+\frac{1}{2}\left(1+n_{1}^{2}\right)\left(n_{3}^{2}-n_{2}^{2}\right) d^{33}+ \\
& -n_{1} n_{2}\left(1-n_{1}^{2}\right) d^{12}-n_{1} n_{3}\left(1-n_{1}^{2}\right) d^{13}+n_{2} n_{3}\left(1+n_{1}^{2}\right) d^{23} \\
c^{33}= & \frac{1}{2}\left(1+n_{3}^{2}\right)\left(n_{1}^{2}-n_{2}^{2}\right) d^{11}+\left(1-\frac{3}{2} n_{3}^{2}+\frac{1}{2} n_{3}^{4}-\frac{1}{2} n_{2}^{2}-\frac{1}{2} n_{2}^{2} n_{3}^{2}\right) d^{33}+ \\
& +n_{1} n_{2}\left(1+n_{3}^{2}\right) d^{12}-n_{1} n_{3}\left(1-n_{3}^{2}\right) d^{13}-n_{2} n_{3}\left(1-n_{3}^{2}\right) d^{23} \\
& c^{22}=-c^{11}-c^{33} \\
& c^{12}=\frac{1}{2} n_{1} n_{2}\left(n_{1}^{2}-n_{2}^{2}\right) d^{11}+n_{1} n_{2}\left(1-\frac{1}{2} n_{2}^{2}+\frac{1}{2} n_{3}^{2}\right) d^{33}+ \\
& +\left(1-n_{1}^{2}\right)\left(1-n_{2}^{2}\right) d^{12}-n_{2} n_{3}\left(1-n_{1}^{2}\right) d^{13}-n_{1} n_{3}\left(1-n_{2}^{2}\right) d^{23} \\
c^{13}= & -n_{1} n_{3}\left(1-\frac{1}{2} n_{1}^{2}+\frac{1}{2} n_{2}^{2}\right) d^{11}-n_{1} n_{3}\left(1+\frac{1}{2} n_{2}^{2}-\frac{1}{2} n_{3}^{2}\right) d^{33}+ \\
- & n_{2} n_{3}\left(1-n_{1}^{2}\right) d^{12}+\left(1-n_{1}^{2}\right)\left(1-n_{3}^{2}\right) d^{13}-n_{1} n_{2}\left(1-n_{3}^{2}\right) d^{23}
\end{aligned}
$$




$$
\begin{aligned}
c^{23} & =n_{2} n_{3}\left(1+\frac{1}{2} n_{1}^{2}-\frac{1}{2} n_{2}^{2}\right) d^{11}-\frac{1}{2} n_{2} n_{3}\left(n_{2}^{2}-n_{3}^{2}\right) d^{33}+ \\
& -n_{1} n_{3}\left(1-n_{2}^{2}\right) d^{12}-n_{1} n_{2}\left(1-n_{3}^{2}\right) d^{13}+\left(1-n_{2}^{2}\right)\left(1-n_{3}^{2}\right) d^{23}
\end{aligned}
$$

[1] Proc. of the first E. Amaldi Conference on Gravitational Wave Experiments (E. Coccia, G. Pizzella, F. Ronga eds., World Scientific, 1995).

[2] Proc. of the International Conference on Gravitational Waves: Sources and detectors (I. Ciufolini and F. Fidecaro eds., World Scientific, 1997).

[3] L. D. Landau and E. M. Lifshitz, Course of Theoretical Physics Vol. 2: The Classical Theory of Fields (Pergamon Press, Oxford, 1975)

[4] P. C. Peters and J. Mathews, Phys. Rev. 131, 435 (1963).

[5] A. G. Wiseman, Phys. Rev. D , 46, 1517 (1992).

[6] X. Zhuge, J. M. Centrella, and S. L. W. McMillan, Phys. Rev. D 50, 6247 (1994).

[7] C. Moreno-Garrido, E. Mediavilla, and J. Buitrago, Mon. Not. Royal Astron. Soc. 274, 115 (1995).

[8] J. Hough et al., in Proc. of the first E. Amaldi Conference on Gravitational Wave Experiments, 50, ed. E. Coccia, G. Pizzella, and F. Ronga (Singapore, World Scientific, 1995).

[9] C. Cutler, gr-qc/9703068

[10] V. M. Lipunov, K. A. Postnov, and M. E. Prokhorov, Astron. and Astroph. 176, L1 (1987).

[11] D. Hils, P. L. Bender, and R. F. Webbink, Astroph. J. 360, 75 (1990).

[12] L. Blanchet, B. R. Iyer, C. M. Will, and A. G. Wiseman, Class. Quant. Grav. 13, 575 (1996).

[13] I. B. Khriplovich and A. A. Pomeransky, Phys. Lett. A 216, 7 (1996).

[14] J. R. Wilson, G. J. Mathews, and P. Marronetti, Phys. Rev. D 54, 1317 (1996).

[15] L. Blanchet, Phys. Rev. D 54, 1417 (1996).

[16] M. Ruffert, H.-Th. Janka, and G. Schäfer, Astron. Astroph. 311, 532 (1996).

[17] T. A. Apostolatos, Phys. Rev. D 54, 2438 (1996).

[18] K. S. Hirata et al., Phys. Rev. Lett. 58, 1490 (1987).

[19] K. S. Hirata et al., Phys. Rev. D 38, 448 (1988).

[20] R. M. Bionta et al., Phys. Rev. Lett. 58, 1494 (1987).

[21] C. B. Bratton et al., Phys. Rev. D 37, 3361 (1988).

[22] C. D. Baylin, E. P. Rubinstein, T. M. Girard, D. I. Dinescu, F. A. Rasio, and B. Yanny, Astrophys. J. Lett. 433, L89 (1994).

[23] S. W. McCuskey, Introduction to Celestial Mechanics (Addison-Wesley, Reading, MS, 1963).

[24] D. Brouwer and G. M. Clemence, Methods of Celestial Mechanics (Academic Press, New York, NY, 1961).

[25] M. Calura, P. Fortini, and E. Montanari, Phys. Rev. D 56, 4782 (1997).

[26] C. Misner, K. S. Thorne, and J. A. Wheeler, Gravitation (Freeman, San Francisco, 1973).

FIG. 1. Amplitude of the term $h_{11}^{(i)}$ of the signal emitted by the triple star system of Section 4.1 ; the orbital plane is perpendicular to the observation direction. For the meaning of the symbols used see eqs. (3.20)- (3.22). a) The fundamental frequency is $N_{i} / \pi$. b) The modulation of the signal has an angular frequency given by the rate of periastron precession $\delta n_{i}$.

FIG. 2. Amplitude of the interaction term $h_{11}^{(i o)}$ emitted by the triple star system of Section 4.1; the orbital plane is perpendicular to the observation direction. For the meaning of the symbols used see eqs. (3.20)- (3.22). a) The fundamental frequency is $N_{i} /(2 \pi)$ while the frequency modulation is $N_{o} /(2 \pi)$. b) The third modulating angular frequency is the periastron precession rate $\delta n_{i}$.

FIG. 3. Amplitude of $h_{11}^{(i o)}+h_{11}^{(i)}$, the detectable signal (see considerations in the text) emitted by the triple star system of Section 4.1. a) It is the sum of figs. $1 \mathrm{a}$ and $2 \mathrm{a}$. b) It is the sum of figs. $1 \mathrm{~b}$ and $2 \mathrm{~b}$. 
FIG. 4. Amplitude of the inner term $h_{11}^{(i)}$ of the gravitational wave emitted by the triple star system of Section 4.2 ; the orbital plane is perpendicular to the observation direction. For the meaning of the symbols used see eqs. (3.20)- (3.22). The fundamental frequency is $N_{i} / \pi$.

FIG. 5. Amplitude of the interaction term $h_{11}^{(i o)}$ emitted by the triple star system of Section 4.2, when the orbital plane is perpendicular to the observation direction. For the meaning of the symbols used see eqs. (3.20)- (3.22). The fundamental frequency is $N_{i} /(2 \pi)$; the modulation is given by $N_{o} /(2 \pi)$.

FIG. 6. Amplitude of $h_{11}^{(i o)}+h_{11}^{(i)}$, the detectable signal (see considerations in the text) emitted by the triple star system of Section 4.2. It is the sum of figs. 4 and 5 . 


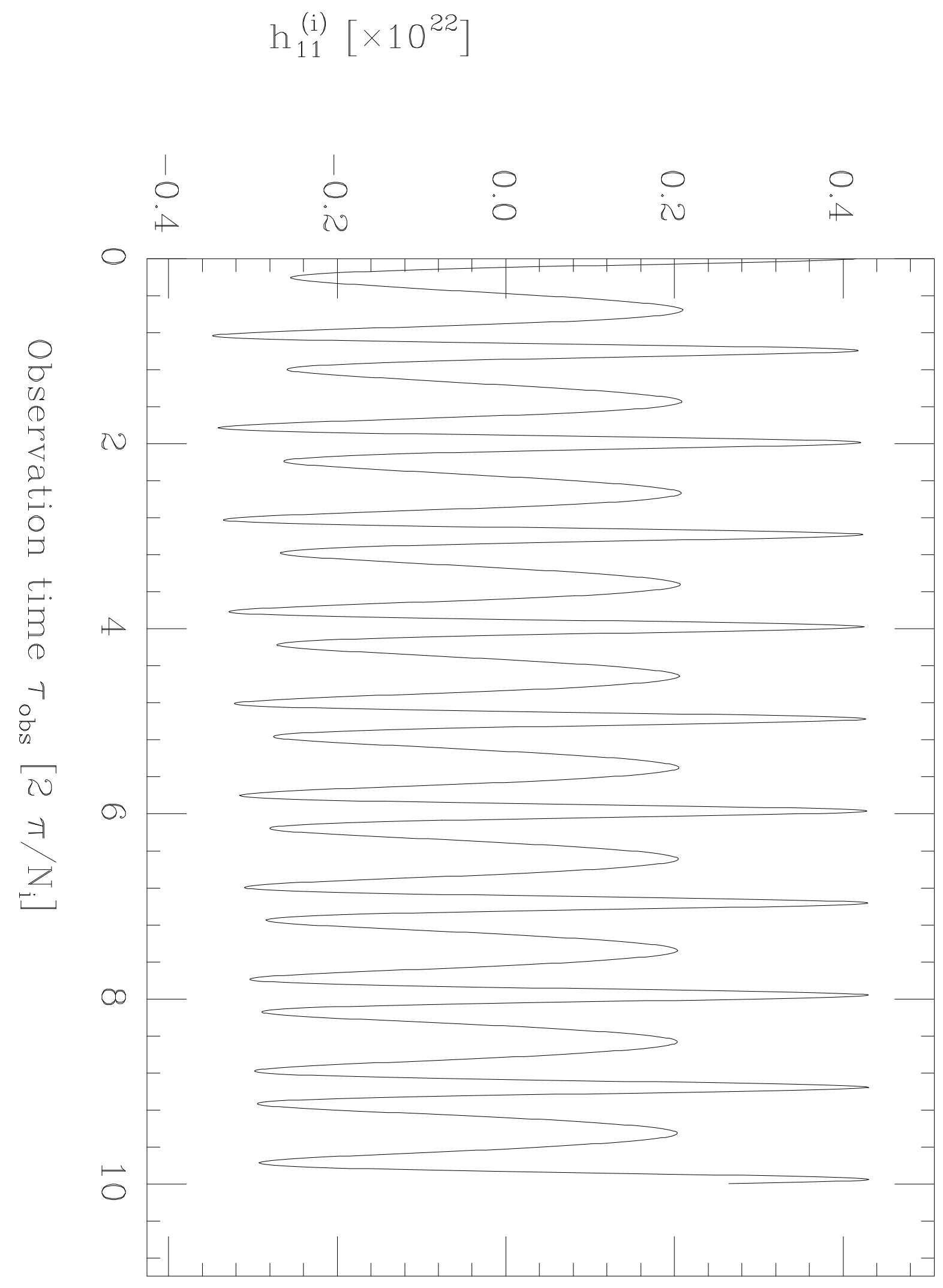


$h_{11}^{(i)}\left[\times 10^{22}\right]$

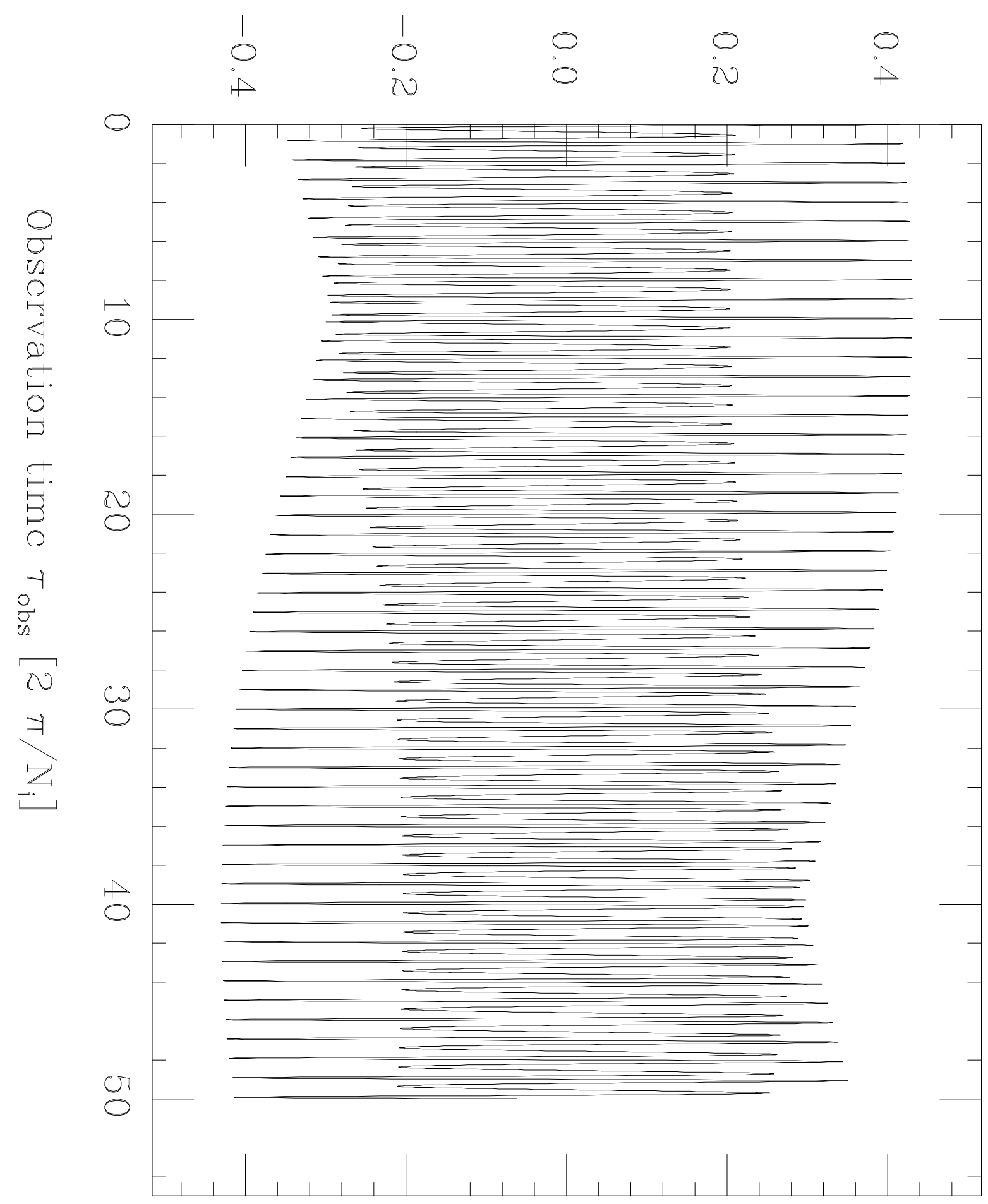


$\mathrm{h}_{11}^{(\text {io) }}\left[\times 10^{22}\right]$

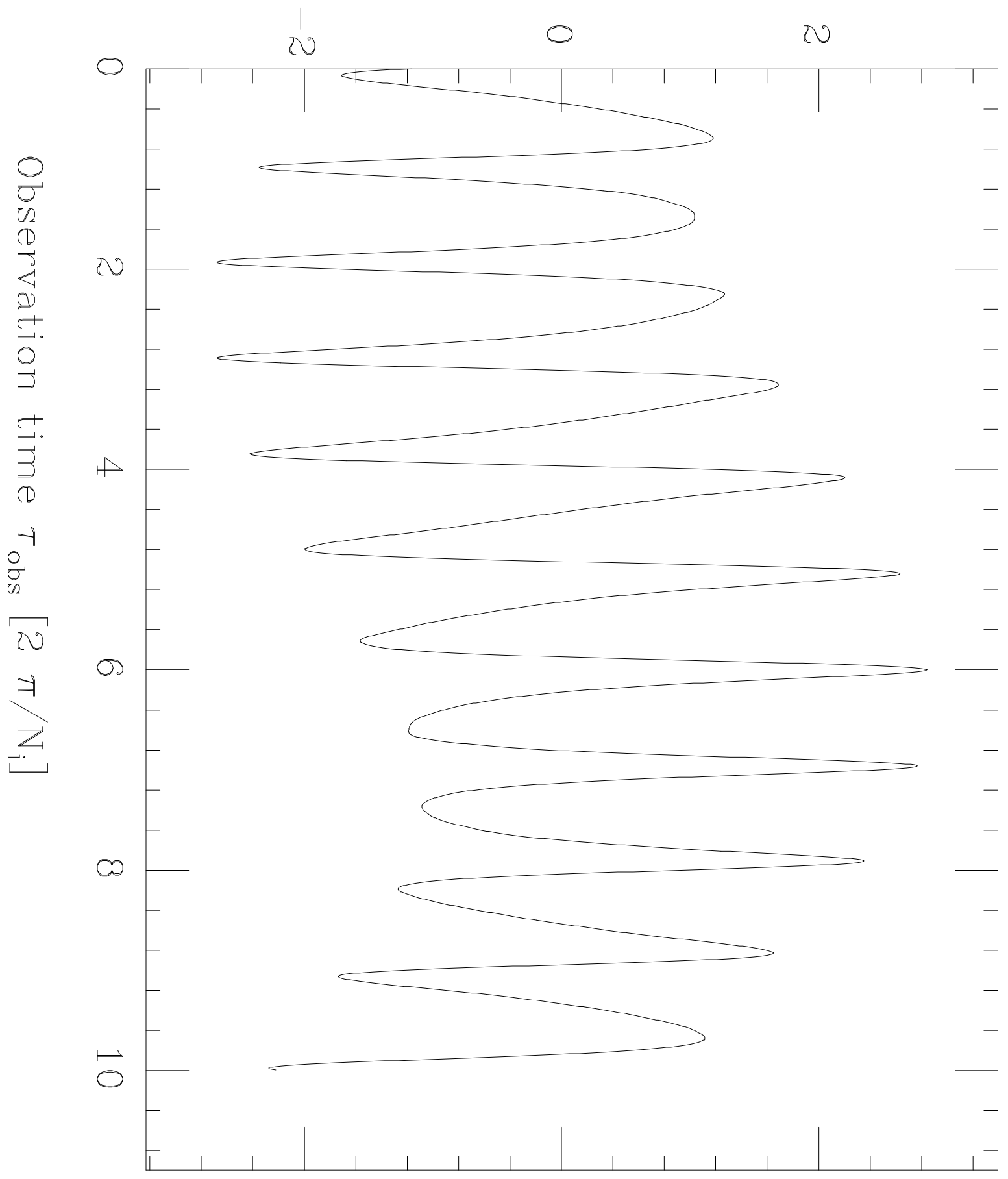


$\mathrm{h}_{11}^{(\text {io })}\left[\times 10^{22}\right]$

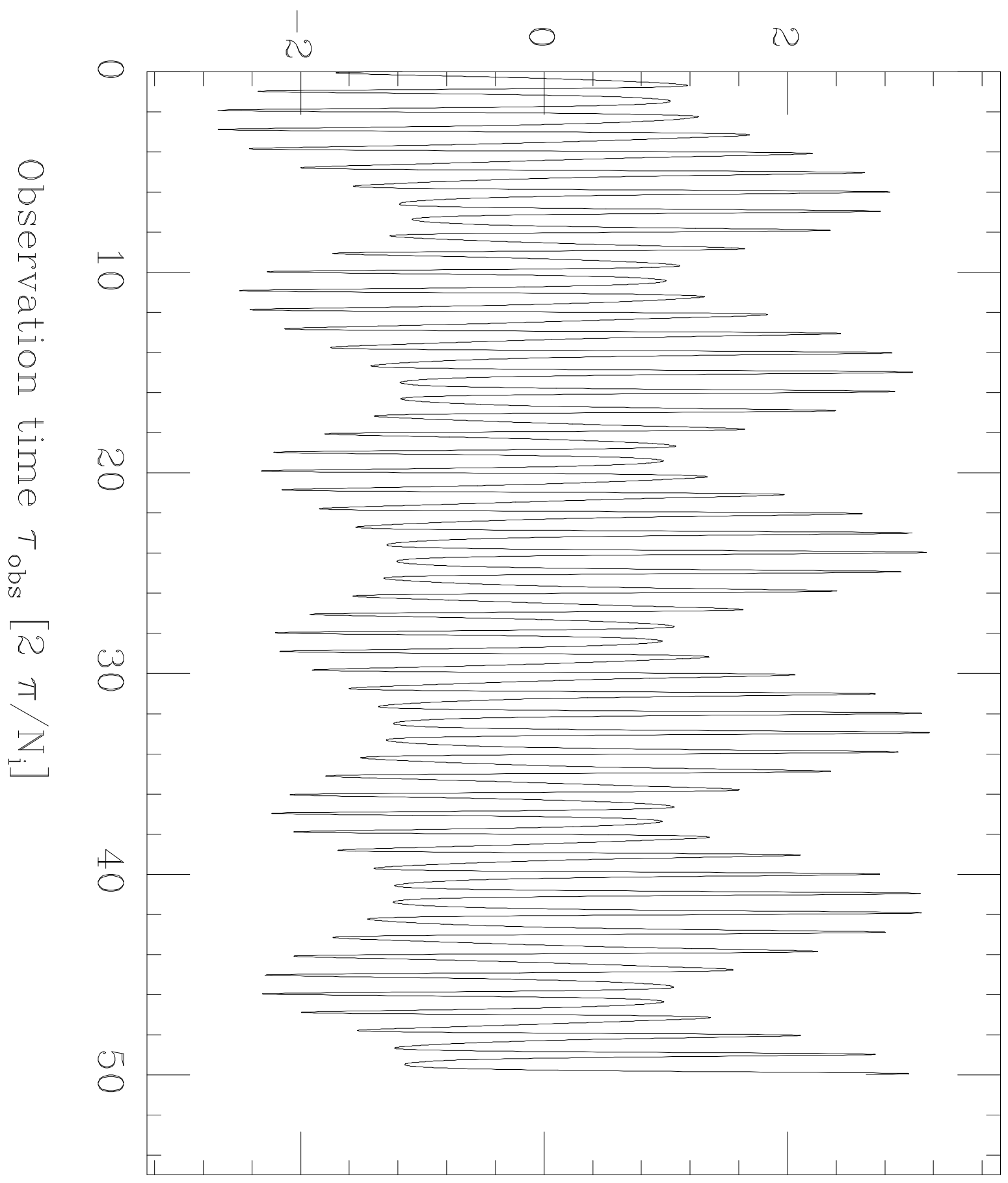


$\mathbf{h}_{11}^{(\mathrm{i})}+\mathbf{h}_{11}^{(\mathrm{io})}\left[\times 10^{22}\right]$

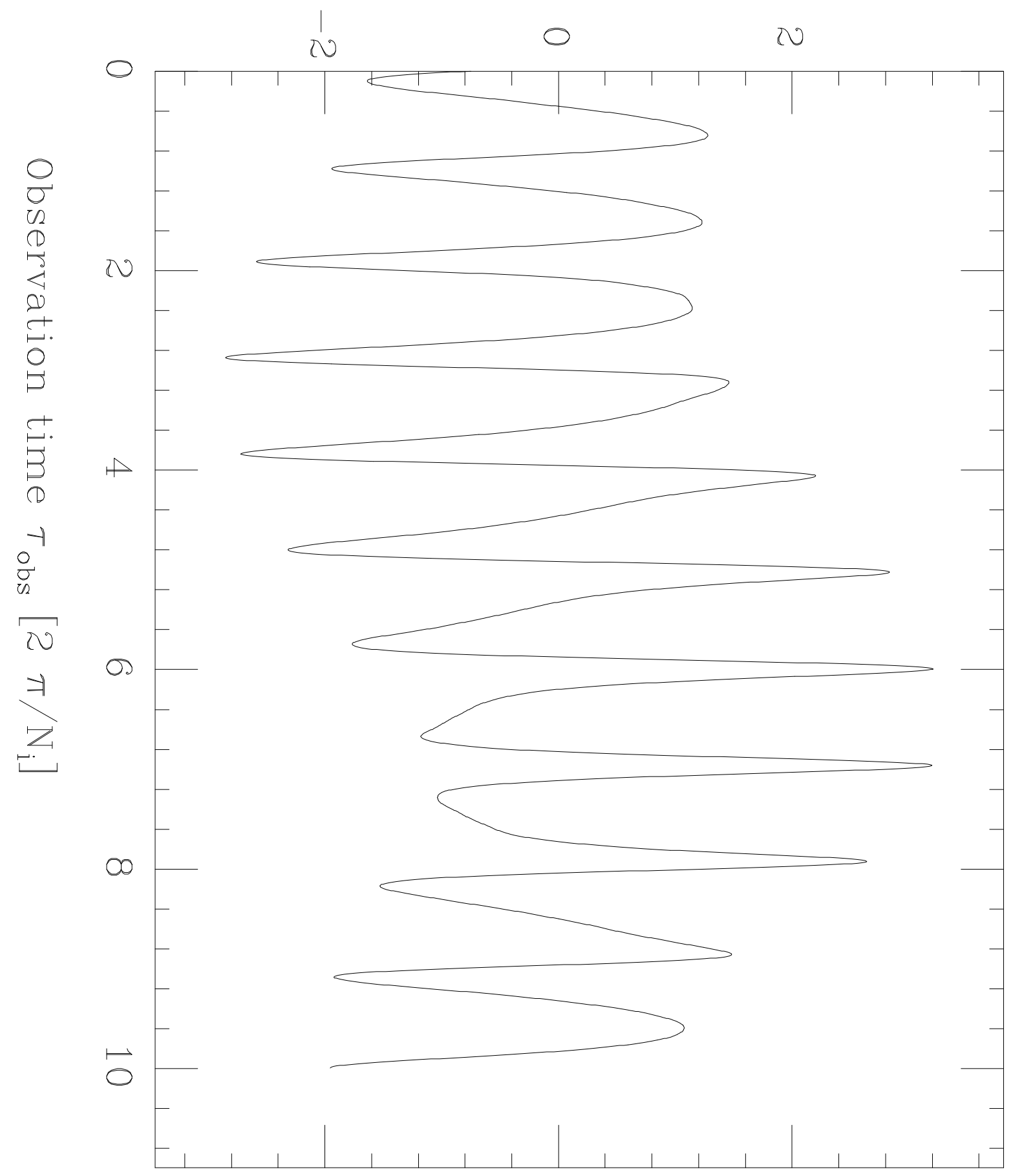


$\mathrm{h}_{11}^{(\mathrm{i})}+\mathrm{h}_{11}^{(\mathrm{io})}\left[\times 10^{22}\right]$

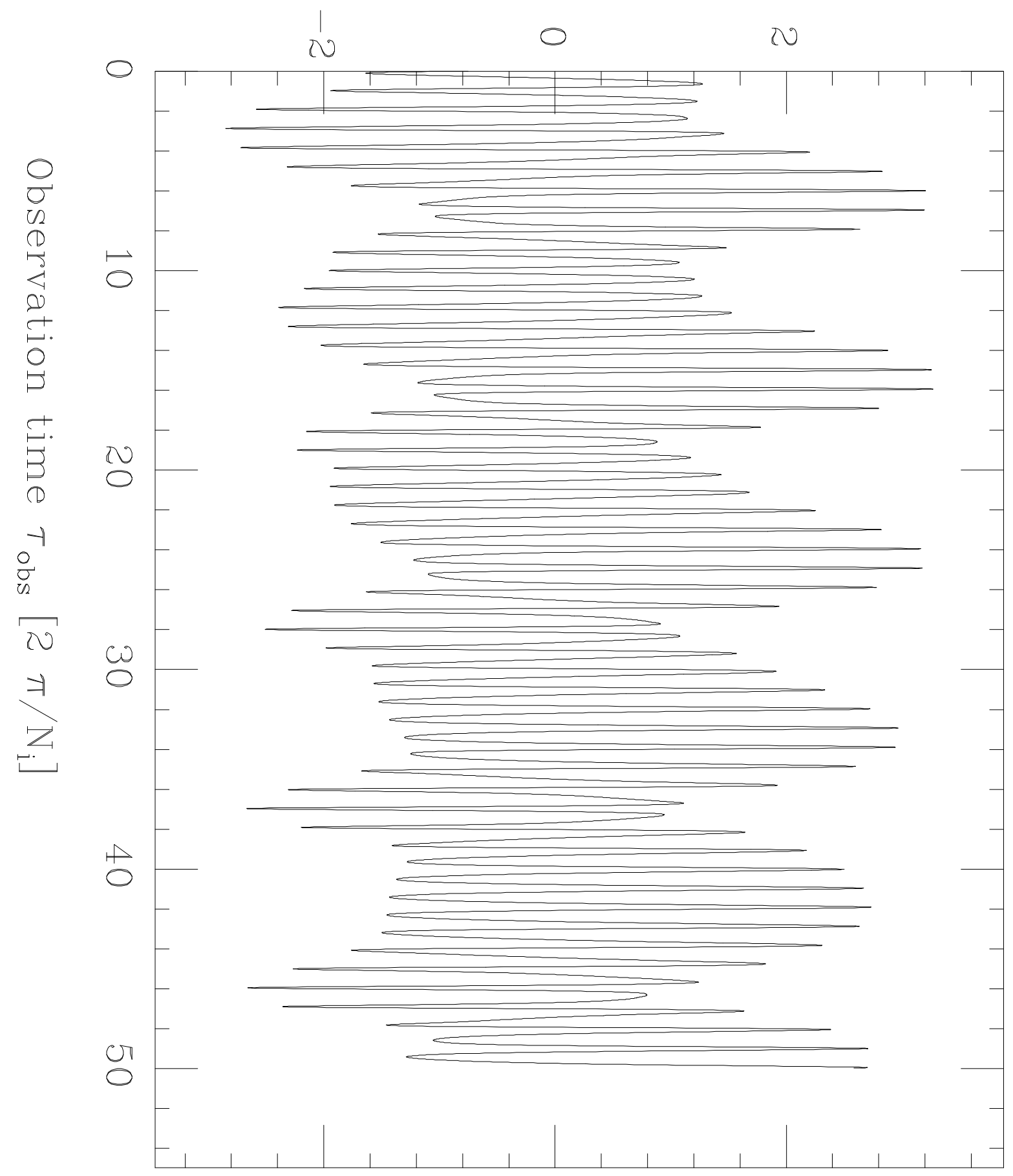




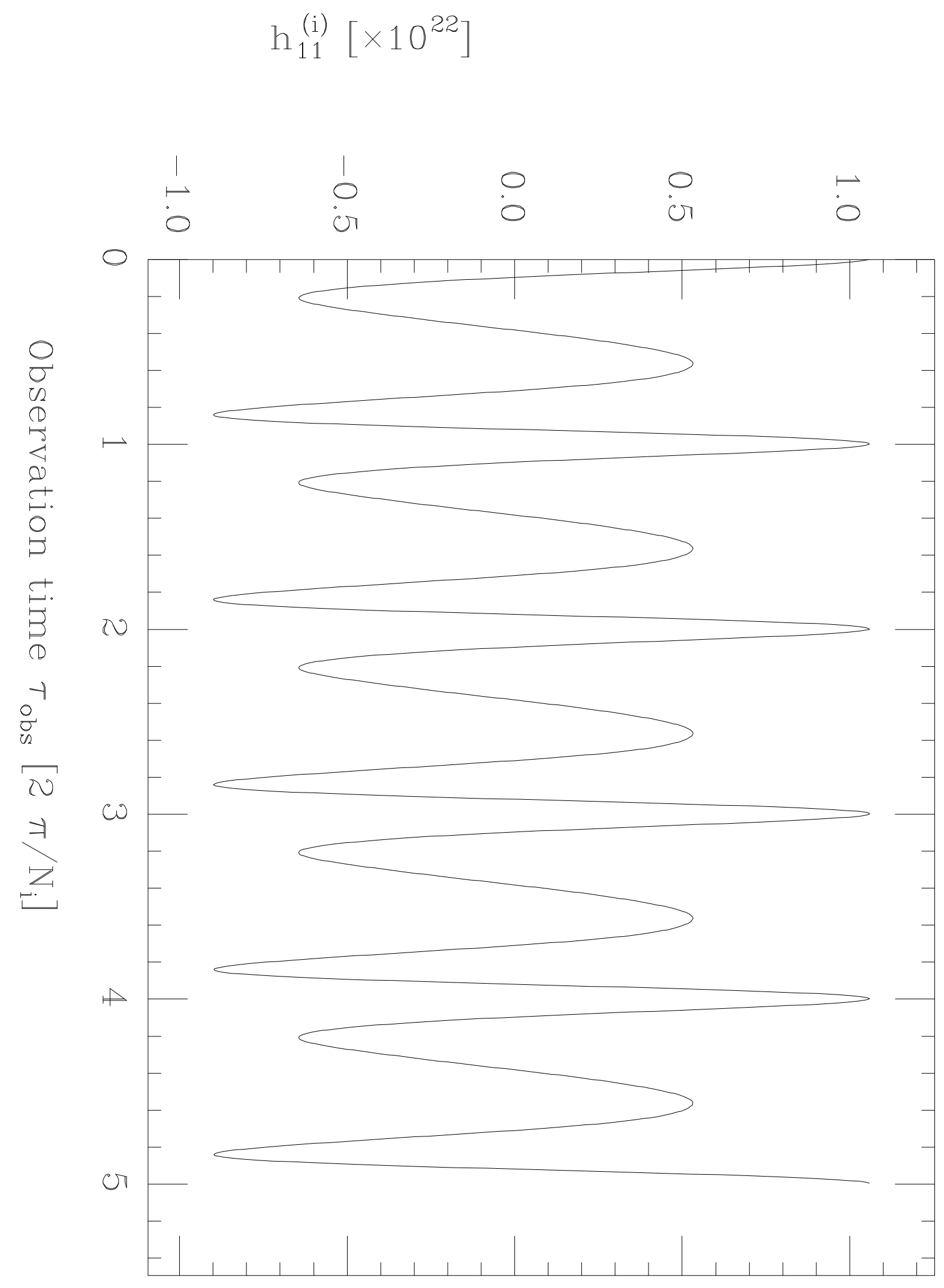


$\mathrm{h}_{11}^{(\text {io) }}\left[\times 10^{22}\right]$

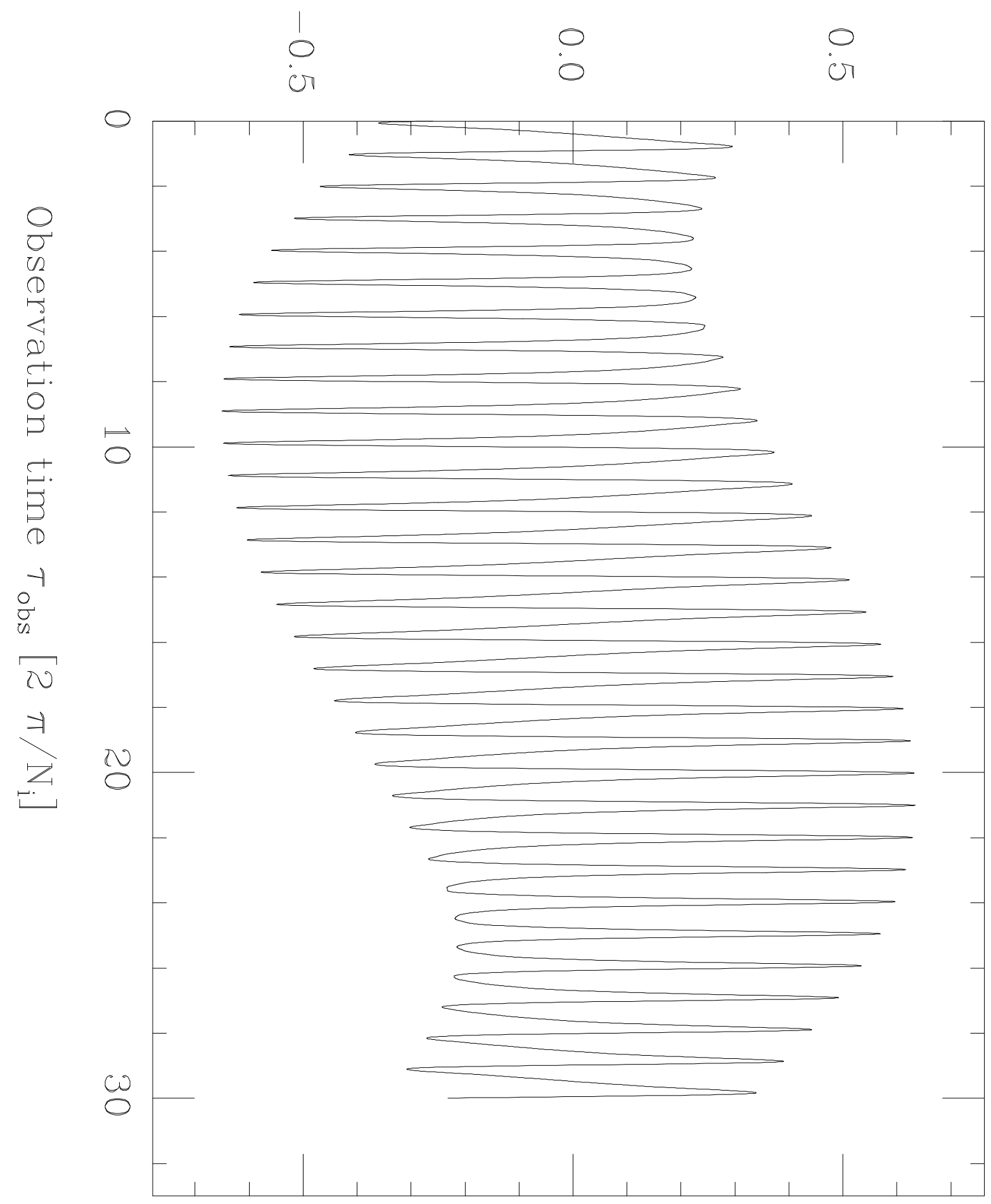


$\mathbf{h}_{11}^{(\mathrm{i})}+\mathrm{h}_{11}^{(\mathrm{io})}\left[\times 10^{22}\right]$

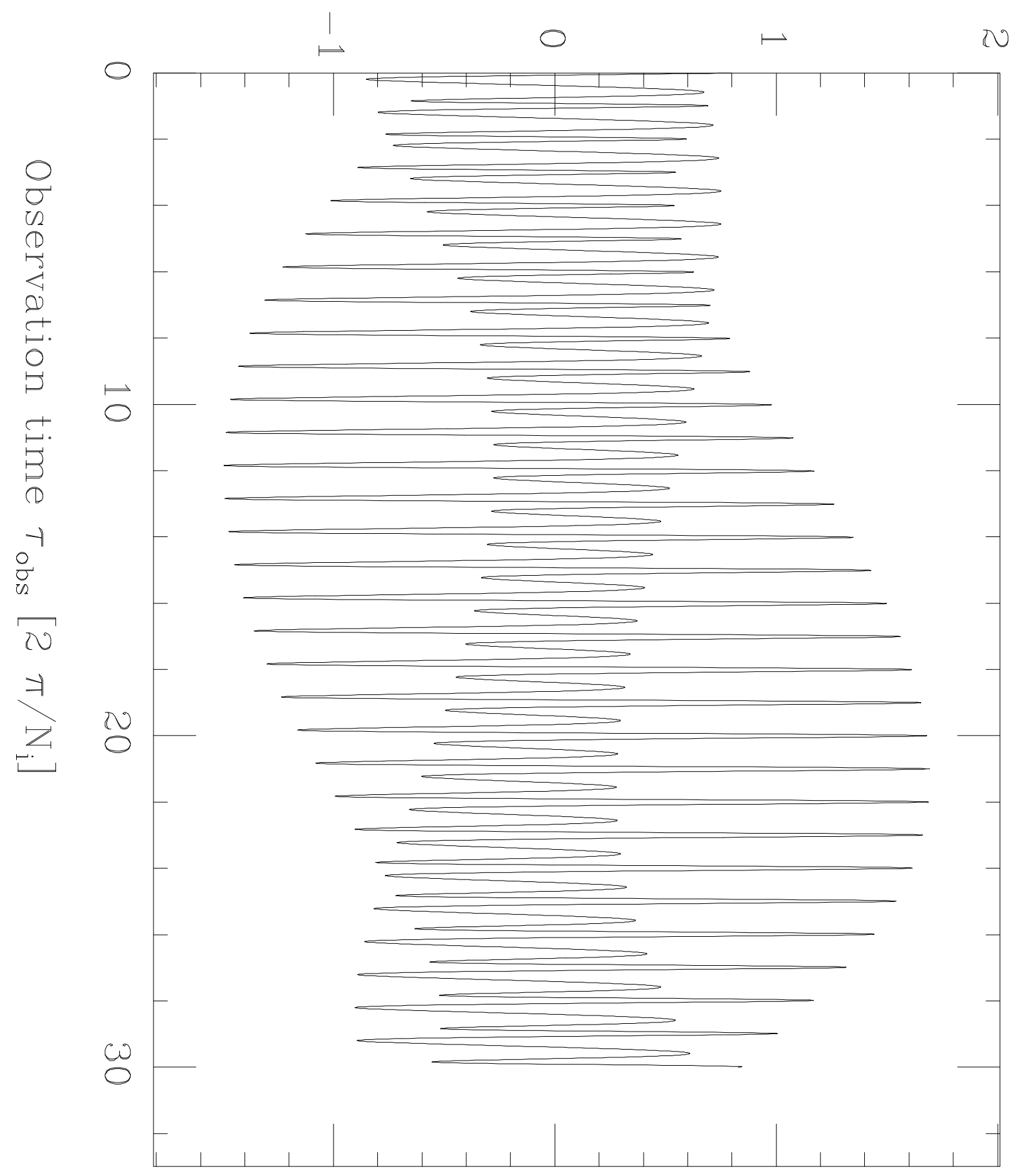

